Monatsschrift f. Geburtshülfe u. Gynäkologie 1932;92:I-IV

\title{
Contents, Vol. 92, 1932
}

Inhaltsverzeichnis.

Originalarbeiten. seite

Baumert, Maria, Aussichten und G-renzen für die Frühdiagnose des

Gebärmutterkrebses 90

Baumgart, Hans, Blasenmole bei õljähriger Frau. Ein Beitrag zur

Fehldiagnose in der Gynäkologie 34

Bickel, L., Tonometrisehe Untersuchungen an Frauen in den ver-

schiedenen G-enerationsphasen. 257

Bromberger, Waiter, Das Schicksal der Kaiserschnittkinder. ... 39 Bud, Georg von, Uber die

Behandlung der Eklampsie auf Grund

1ljähriger Erfahrungen 11

Bychowskaja, A. S., Einige klinische Beobachtungen über die Ery-

throzyten-Senkungsreaktion in der Gynäkologie 373

Eldering, Bram, Uber die Verlagerung des Zökums in der Schwanger-

schaft 377

Bufinger, Heinrich, Die Glykoregulation der Plazenta im Verlaufe

der Schwangerschaft 272

Fels, Erich, Hormonverhältnisse bei einem Steißteratom

Flessa, W., Der .Kristellersche Handgriff ... 25

Fraymann, S., Über einige Möglichkeiten des Einwirkens per rectum

auf den Geburtsverlauf

Gaeßler, E. O., Beeinflussen die Hypophysenvorderlappenpräparate des

Schwangerenharns den Ruhegrundumsatz und die speziíisch-dyna-

mische Eiweißwirkung? 397

Garfunkel, P., siehe Schiwowa, B.

Glas, Rudolf, Über klimakterische Beschwerden nach gynäkologischen

Operationen . . 409

Halber, W., siehe Rosenblatt, J.

Hochloff, A. W., .Beitrag zur Hämatologie des Menstrualblutes . . 269

Jacoby, Irma, Über das Verhalten der Temperatur während des

mensuellen Zyklus ... 337

Itzkin, S., Die akute Herdinfektion, insbesondere die dentale in der

Geburtshilfe.

68

Kückens, H., Über die Kombination vonTuberkulose undGeschwülsten

im weiblichen Genitalapparat 58

Liebisch, Gerhard, Abriß der kleinen Schamlippe bei spontaner

G.eburt $\quad 190$

Moschkow, B. N., Das Hämatom der Geburtswege 421

Muck, 0., Feststellungen bei der puerperalen Präeklampsie und ver- 
wandten angiospastischen Zuständen durch den Adrenalin-Sondenversuch 368

Ochssée, M. Boon von, und A. Tholen, Zur Einnistung des menschlichen Eies in die Uterusschleimhaut . . 178

Ostermann, Wilhelm, Mischgeschwulst der Leber bei einem Neu-

geborenen 191

Pruszczynski, A., siehe Rosenblatt, J.

Rosenblatt, J., W. Halber und A. Pruszczynski, Die patho-

logisch-anatomischen Veränderungen im Eierstock nach protrahierten

Vorderlappenliormoneinverleibungen und ihre eventuellen Folgen . 284

- Berichti gung 464

Rosenstein, W., Indikation und Technik der Kolpokleisis bei karzi-

nomatösen Fisteln _..... 296

Rubin, I. C, Die Rolle der Appendizitis in der Ätiologie der weiblichen

Bterilität 161

Schapiro, J., siehe Schiwo $\Lambda v a, B$.

Schiwowa, B., P. Garfunkel und J. Schapiro, Über primäre und

wiederholte Tubargravidität 292

IV

Inhaltsverzeichnis.

Seit $\beta$ Schor, M. I., siehe Ts chert ok, R. A. Sdrawomyslow, W. I., Moorbäder, kombiniert mit parenteraler Ich-

thyolbehandlung bei gynäkologiscben Erkrankungen

Seitz, L., Über Genese, Klinik und Therapie der Edometriosis .... 1 Stefancsik, Sz, Bciträge zur Kasuistik der Granulosazellentumoren . 400 Tholen, A., siehe Ochssée, M. Boon von. Ts chert ok, R. A., und M. I. Schor, Tubendurchgängigkeit naeh

intrauteriner Jodinjektion 186

Vaubel, Hildegard, F.rauensport und Gesundheit 173

Wenzkowski, M. K., Über Verletzungen der weiblichen Genitalien

sub coitu 438

Will, Rudolf, Über die Zangengeburten an der Universi†äts-Frauen-

klinik Leipzig in der Zeit vom 1. Januar 1925 bis 31. Dezember 19ki9 347

LudwigGummert $\uparrow \quad 336$

OttomarHoehne† 332

Paul Eißmann $\dagger \quad 159$

Grenzgebíeíe der Geburtshilfe und Gynäkologie.

Soziale Gynäkologie und Geburtshilfe. Von Fr. Chr. Geller-Breslau . 112

Konstitutionstherapie. Von A. Hermstein-Breslau 121

Physiologie und Krankheiten der Neugeborenen einschließlich derjenigen

der weiblichen Brust. (Jahresbericht 11.) Von Privatdozent H. Rossen-

be ck-Gießen 197

Tuberkulose. Von Prof. Dr. K. Hansen und Prof. Dr. F. Schultze-

Rhonhof-Heidelberg 301

Psychiatrie, Psychotherapie und Neurologie. Von Prof. Dr. Heinz

Küstner-Leipzig $\quad 448$

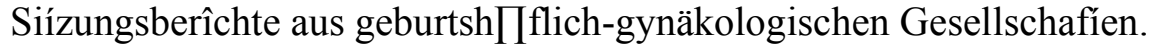


Gynäkologische Gesellschaft zu Breslau. Sitzung vom 19. April 1932 . . 127 - $\quad$ Sitzung vom 21. Juni $1932 \quad 318$

Gesellschaft für Geburtshilfe und Gynäkologie zu Berlin. Sitzungen vom

26. Februar und 11. Mai 1932

136

Sitzungen vom 29. April und 27. Mai 1932

231

Sitzung vom 10. Juni 1932.

325

Sitzungen vom 8. und 15. Juli $1932 \quad 456$

Der Ausflug nach Müncheberg am 16. Juli 1932

Mittelrheiniache Gesellschaft für Geburtshilfe und Gynäkologie. 87. Sitzung am 13. Dezember 1931 in Frankfurt a. M 237

Nordwestdeutsche Gesellschaft für Gynäkologie in Göttingen. Sitzungen am 30. April und 1. Mai 1932244

Streiflichter von der 23. Tagung der Deutschen Röntgengesellschaft am 3. bis 5. April 1932 in Dresden. Von Dr. G. H. Schneider-Branden burg a. d. H 154

Geburtshilflich-gynäkologische Gesellschaft in Wien. Sitzungen vom 24. Mai und 14. Juni 1932134

Bericht über den Kongreß der Geburtshelfer und Gynäkologen französischer Zunge am 2. und 3. Oktober 1931 in Bordeaux. Von Dr. H.

Vignes -Paris 150

Buchbesprechungen 155, 462

Personalien und Tagesnachrichten 159, 256, 464 